\title{
Teamwork, Cooperation, Innovation: Clues in the Canadian Success of Stroke Care
}

Can. J. Neurol. Sci. 2009; 36: 133-134

"The man who gets the most satisfactory results is not always the man with the most brilliant single mind, but rather the man who can best coordinate the brains and talents of his associates".

\section{William Alton Jones}

Stroke is a major health issue for patients, families and for the health care system. The physical, social and economic consequences after stroke are even more noticeable in young adults, vital contributors to the workforce.

Stroke in young adults has unique characteristics. For example, the etiologies of stroke are more heterogenous than for older stroke patients who are more likely to have the traditional vascular risk factors. ${ }^{1}$ Moreover, extensive investigations are usually necessary to aid medical decision-making. Despite a thorough evaluation, the etiology may remain undetermined in up to one third of these patients. ${ }^{2}$

In the last decade, different initiatives have been implemented to improve access to specialized care and delivery of services. To date, however, limited information is available on thrombolytic therapy in young adults with stroke. ${ }^{3}$

In this issue of the Journal, Poppe et $\mathrm{al}^{4}$ report a subgroup analysis of patients with ischemic stroke younger than 50 years receiving thrombolytic therapy. This is based on a large, prospective, national study that helped to get IV-t-PA approved in Canada (Canadian Alteplase for Stroke Effectiveness Study CASES). ${ }^{5}$ Main findings include: younger patients had a less severe stroke (median NIHSS 13 vs. $15 ; \mathrm{p}<0.001$ ), and a better survival (10.2 mortality vs $23.5 \%$; $=0.002)$. Yet interestingly, age younger than 50 years was not an independent predictor of favourable 90 -day outcome (mRS score $0-1$ ) or decreased risk of intracranial bleeding. These results were adjusted by gender, baseline NIHSS and glucose level. Of note, baseline blood pressure has been shown to affect stroke outcome. Readers may wonder whether these results would change if blood pressure has been included in the adjustment. There was a significant difference in pretreatment systolic blood pressure- mean in systolic blood pressure was $13.8 \mathrm{mmHg}$ lower in the younger group. In addition, this is a non-controlled open-label study and investigators were able to select patients to participate in CASES.

According to Heart and Stroke Foundation Canada, the number of Canadians living with some form of heart disease or stroke (based on self report) is $1.3 \%$ for those age 20-49 years (Heart and Stroke Foundation of Canada - Tipping the Scales of Progress: Heart Disease and Stroke in Canada, 2006). In a large study including over 26,000 patients hospitalized with an ischemic stroke across Canada, $5.2 \%$ were 50 years or younger. ${ }^{6}$
Unpublished data from the Registry of the Canadian Stroke network showed no significant age differences in thrombolysis rate $(14 \%$ for younger than $59,14.7 \%$ for those aged $60-79$ and $13.1 \%$ for those over $80 ; \mathrm{p}=0.69$ ). In the National Institute of Neurologic Disorders and Stroke (NINDS) t-PA trial combined, 67 patients were 50 years of age or less $(11 \%), 89$ were ages $51-$ $60(14 \%), 302$ were $61-75(48 \%)$ and 166 were over 75 years of age $(27 \%) .{ }^{7}$ Age is the second most important predictor of stroke outcome (following stroke severity), yet the original publications from other large randomized clinical trials (ECASS I, II and III, ATLANTIS I and II, PROACT II $)^{8-11}$ or community studies (SITS-MOST) $^{12}$ on thrombolysis for acute stroke don't report baseline characteristics or results stratified by age.

What can we learn from this study? First, CASES represent a large cooperative initiative from physicians across the country to study the use of intravenous rtPA in Canada. It included more young individuals with strokes than the NINDS trial (99 vs. 67 patients younger than 50). 5 Second, this study confirms previous findings that younger patients have lower comorbidities, less severe strokes, lower length of hospitalizations and better survival.

Third, according to this report, younger age doesn't appear to be a predictor of lower disability or risk of $\mathrm{ICH}$. Intuitively, we may expect a better outcome in younger individuals. The small sample size may explain, at least in part, the reported findings (Table 4).

Fourth, there were no significant age disparities in parameters of processes of care (door-to-CT, door-to-needle times, proportion of patients treated within 120 min of onset, etc) between younger and older than 50 years.

Finally, CASES represents another example on how a mutual effort can bring an educational, scientific, and patient-care benefit in Canada. Other effective initiatives include groups of stroke investigators who work together with the several companies to optimize the implementation clinical trials (Canadian Stroke Consortium), to conduct epidemiological and health services stroke research across Canada [Stroke Outcome Research Canada (SORCan) Working group- www.sorcan.ca, Canadian Stroke Network]. The Canadian Stroke Strategy aims to bring organized stroke care to all Canadian provinces, and the Canadian Stroke Network, a National network of excellence, has stimulated stroke research from molecules to healthcare system change.

\section{Future directions}

Since the approval of intravenous thrombolysis almost ten years ago, there have been new developments. Recently, we learned that the time window could be expanded to 4.5 hours. ${ }^{11}$ 
Medical devices for clot removal/lysis or combined procedures (IV + IA thrombolysis) have shown promising results. Although new therapeutic interventions may be available for selected candidates (irrespective of the age), young adults are likely to achieve the greatest benefit. The number of published research studies in stroke has dramatically increased; for example, according to a MEDLINE search there were 3798 papers on stroke published from 1980-1990, 10,333 in 1990-2000 and 20,446 in 2000-2008.

The nihilistic era in the approach to stroke care has ended. The future is here. In Canada, a successful model of national and international cooperation among stroke investigators is evolving and expanding with the goal of developing the better treatments and better outcomes from this common life-threatening and disabling disorder that afflicts the young as well as old segments of the population.
Gustavo Saposnik St. Michael's Hospital, University of Toronto Toronto, Ontario saposnikg@smh.toronto.on.ca

\section{REFERENCES}

1. Bogousslavsky J, Pierre P. Ischemic stroke in patients under age 45. Neurol Clin. 1992 Feb;10(1):113-24

2. Adams HP, Jr., del Zoppo G, Alberts MJ, Bhatt DL, Brass L, Furlan A, et al. Guidelines for the early management of adults with ischemic stroke: a guideline from the American Heart Association/American Stroke Association Stroke Council, Clinical Cardiology Council, Cardiovascular Radiology and Intervention Council, and the Atherosclerotic Peripheral Vascular Disease and Quality of Care Outcomes in Research Interdisciplinary Working Groups: the American Academy of Neurology affirms the value of this guideline as an educational tool for neurologists. Stroke. 2007 May;38(5):1655-711.

3. Wagner JC, Lutsep HL. Thrombolysis in young adults. J Thromb Thrombolysis. 2005 Oct;20(2):133-6.

4. Poppe A, Hill M, AM B. Intravenous thrombolysis for acute ischemic stroke in young adult patients. Can J Neurol Sci. 2009; 36:161-7.

5. Hill MD, Buchan AM. Thrombolysis for acute ischemic stroke: results of the Canadian Alteplase for Stroke Effectiveness Study. CMAJ. 2005 May 10;172(10):1307-12.

6. O'Donnell MJ, Kapral MK, Fang J, Saposnik G, Eikelboom JW, Oczkowski W, et al. Gastrointestinal bleeding after acute ischemic stroke. Neurology. 2008 Aug 26;71(9):650-5.

7. Tissue plasminogen activator for acute ischemic stroke. The National Institute of Neurological Disorders and Stroke rt-PA Stroke Study Group. N Engl J Med. 1995 Dec 14;333(24): 1581-7.

8. Ringleb PA, Schellinger PD, Schranz C, Hacke W. Thrombolytic therapy within 3 to 6 hours after onset of ischemic stroke: useful or harmful? Stroke. 2002 May;33(5):1437-41.

9. Fiorelli M, Bastianello S, von Kummer R, et al. Hemorrhagic transformation within 36 hours of a cerebral infarct: relationships with early clinical deterioration and 3-month outcome in the European Cooperative Acute Stroke Study I (ECASS I) cohort. Stroke. 1999 Nov;30(11):2280-4.

10. Clark WM, Wissman S, Albers GW, Jhamandas JH, Madden KP, Hamilton S. Recombinant tissue-type plasminogen activator (Alteplase) for ischemic stroke 3 to 5 hours after symptom onset. The ATLANTIS Study: a randomized controlled trial. Alteplase Thrombolysis for Acute Noninterventional Therapy in Ischemic Stroke. Jama. 1999 Dec 1;282(21):2019-26.

11. Hacke W, Kaste M, Bluhmki E, Brozman, M, Davalos, A, Guidetti $\mathrm{D}$, et al. Thrombolysis with alteplase 3 to 4.5 hours after acute ischemic stroke. N Engl J Med. 2008 Sep 25;359(13): 1317-29.

12. Wahlgren N, Ahmed N, Davalos A, Hacke W, Milan M, Muir K, et al. Thrombolysis with alteplase $3-4.5 \mathrm{~h}$ after acute ischaemic stroke (SITS-ISTR): an observational study. Lancet. 2008 Oct 11;372(9646):1303-9. 